\title{
Application of combined techniques for studying nano-minerals in geological system
}

\author{
Seungyeol Lee and Huifang Xu (Lee572@,wisc.edu)
}

Department of Geoscience, University of Wisconsin-Madison, 1215 W. Dayton St., Madison, Wisconsin 53706, United States

High temperature oxidation and fast cooling of basaltic lava resulted in formation of nano-minerals of Fe(III)-based oxides. Modern technology allows us to detect, characterize, and understand the nature of nano-minerals. In the case of macroscopic minerals, X-ray diffraction is useful tool to study their structure from the Bragg peak positions and intensities. However, this is not realistic for nano-minerals, which show broad and continuous intensity distribution that is not amenable to a crystallographic structure solution. Especially, it is challenging to determine its structure at the nanoscale when structures include the short-range ordering, defects, and local domain.

We used integrated techniques (powder diffraction, TEM, Z-contrast imaging and $a b$ initio calculations) for solving this problem and applied some nano-minerals in geological environments. One example is that a natural $\varepsilon-\mathrm{Fe}_{2} \mathrm{O}_{3}$ nano-mineral (luogufengite) that was discovered as nano-crystals on vesicles' surfaces of scoria from Menan Volcanic Complex, Idaho by using combined method of synchrotron powder diffraction and TEM. The luogufengite is an intermediate phase between maghemite and hematite. The crystal size of luogufengite ranges from $\sim 10$ to $120 \mathrm{~nm}$. There are several magnetic nano-minerals in the scoria due to oxidation of basaltic lava at high temperature. We studied the crystal structure, composition, and mineral association of luogufengite. The luogufengite can be promising minerals for understanding paleomagnetism of volcanic rocks due to its large magnetic coercivity for preserving the original planetary magnetic field. The results suggest combined techniques of powder diffraction, TEM, Z-contrast imaging and ab-initio calculations are promising approaches for understanding nanominerals. The combined method will be helpful for broadening our view on roles of nanominerals in the Earth's surface systems. 